\title{
A property of Cesàro-Perron integrals
}

\author{
By L. S. Bosanquet.
}

(Received 19th January, 1940. Read 3rd̀ February, 1940.)

1. It is well known ${ }^{1}$ that if $f(t)$ is (a) integrable in the Lebesgue sense, or more generally $(b)$ integrable in the Perron sense, over every interval $(a, \beta)$ interior to $(a, b)$, and if

$$
\lim _{\substack{a \rightarrow a+0 \\ \beta \rightarrow b-0}} \int_{a}^{\beta} f(t) d t
$$

exists, then $f(t)$ is integrable in the Perron sense over $(a, b)$ to the value $(1 \cdot 1)$.

The first result expresses the fact that a function integrable in the Cauchy-Lebesgue sense is also integrable in the Perron sense, while the second is fundamental in the proof ${ }^{2}$ of the equivalence of the Special Denjoy and Perron integrals. These results have been extended by Miss M. E. Grimshaw ${ }^{3}$ to the case when Perron integrability is replaced by Cesàro-Perron integrability of unit order ${ }^{4}$ and the limits by Cesàro limits. Miss Grimshaw's theorem and its generalisation for Cesàro-Perron integrals of any positive order ${ }^{5}$ have important applications. The object of this note is to prove the general case $^{6}$.

2. Definition. If $\lambda>0$ we say that $f(t) \rightarrow l(C, \lambda)$ as $t \rightarrow a$, or that $C_{\lambda}-\lim _{t \rightarrow a} f(t)=l$, if (i) $f(t)$ is integrable $C_{\mu} P$ in an interval $(a-\eta, a+\eta)$, where $\mu=\max (\lambda-1,0)$, and (ii)

$$
\begin{gathered}
C_{\lambda}\left(f^{\prime}, a, a+h\right)=\frac{\lambda}{h} \int_{0}^{h}\left(1-\frac{u}{h}\right)^{\lambda-1} f(a+u) d u \\
\left(C_{\mu} P\right)
\end{gathered}
$$

tends to $l$ as $h \rightarrow 0$. The usual related notation is defined in the obvious way.

1 See Saks, 8, 247 .

$\because$ See Saks, 8, 247-252.

${ }^{3}$ Grimshaw, 7.

* Burkill, 3.

5 Burkill, 3, 4, 6.

"I am indebted to Dr John Todd who, after I had proved Theorem A, drew my attention to Miss Grimshaw's paper. 
Theorem A. If $\lambda \geqq 0$ and (i) $f(t)$ is integrable $C_{\lambda} P$ in $(a, \beta)$ for every $a, \beta$ such that $a<a<\beta<b$, (ii)

$$
\begin{gathered}
\mathrm{C}_{\lambda}-\lim _{\substack{a \rightarrow a+0 \\
B \rightarrow b-0}} \int_{a}^{\beta} f(t) d t \\
\left(C_{\lambda} P\right)
\end{gathered}
$$

exisls, then $f(t)$ is integrable $C_{\lambda} P$ in $(a, b)$ to the value (2.2).

Proof. We need only consider the case $\lambda>0$. Let

$$
\begin{aligned}
& F(x)=C_{\substack{\lambda \\
a \rightarrow a+0}} \int_{a}^{x} f(t) d t \quad(a<x<b) \\
& \left.F(a)=0 \quad C_{\lambda} P\right) \\
& F(b)=C_{\lambda}-\lim _{x \rightarrow b-0} F(x) .
\end{aligned}
$$

We shall show that, if $\epsilon$ is a given positive number, there is a function $M(x)$ such that

and, for ${ }^{1} a \leqq x \leqq b$,

$$
\text { (1) } M(a)=0 \text {, }
$$

(2) $M(x)$ is $C_{\lambda}$-continuous ${ }^{2}$,

(3) $0 \leqq M(x)-F(x)<\epsilon$,

(4) $C_{\lambda} D_{*} M(x) \geqq f(x)$,

(5) $C_{\lambda} D_{*} M(x)>-\infty$,

where

$$
C_{\lambda} D_{*} M(x)=\lim _{h \rightarrow 0} \frac{C_{\lambda}(M, x, x+h)-M(x)}{l_{b} /(\lambda+1)} .
$$

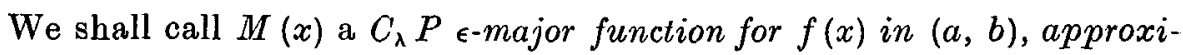
mating to $F(x)$.

A similar argument will show the existence of a $C_{\lambda} P \quad \epsilon$-minor function $m(x)$ for $f(x)$ in $(a, b)$, approximating to $F^{\prime}(x)$.

This will show that $f(t)$ is integrable $C_{\lambda} P$ in $(a, b)$, and that, for $a \leqq x \leqq b$,

$$
\left(C_{\lambda} P\right) \int_{a}^{x} f(t) d t=F(x)
$$

${ }^{x}$ It is to be understood that conditions (2), (4) and (5) need only hold in a one-sided sense at $x=a$ and $x=b$.

2 i.e. $O_{\lambda}(M, x, x+h) \rightarrow M(x)$ as $h \rightarrow 0$. 
Let numbers $x_{n}$ be chosen so that

$$
\begin{aligned}
\text { (i) } & a<\ldots<x_{-2}<x_{-1}<x_{0}<x_{1}<x_{2}<\ldots<b \text {, } \\
\text { (ii) } & x_{n} \rightarrow a \text { as } n \rightarrow-\infty, \\
\text { (iii) } & x_{n} \rightarrow b \text { as } n \rightarrow \infty .
\end{aligned}
$$

Let $M_{n}(x)$ be a $C_{\lambda} P 2^{-|n|-4} \epsilon$-major function for $f(x)$ in $\left(x_{n-1}, x_{n}\right)$, approximating to $F(x)-F\left(x_{n-1}\right)$, and write

$$
D_{n}(x)=M_{n}(x)-F(x)+F\left(x_{n-1}\right)
$$

Let

$$
\begin{aligned}
& M_{0}(x)=F(x)+\sum_{\nu=-\infty}^{n-1} D_{\nu}\left(x_{\nu}\right)+D_{n}(x),\left(\begin{array}{l}
x_{n-1} \leqq x<x_{n}, \\
-\infty<n<\infty
\end{array}\right), \\
& M_{0}(a)=0, \\
& M_{0}(b)=F(b)+\sum_{\nu=-\infty}^{\infty} D_{\nu}\left(x_{\nu}\right) .
\end{aligned}
$$

Then $M_{0}(x)$ is $C_{\lambda}$-continuous in $a<x<b$. Also, if $n \leqq 0$ and $x_{n-1} \leqq x<x_{n}$,

$$
0 \leqq M I_{0}(x)-F(x)<\sum_{\nu=-\infty}^{n} 2^{-|\nu|-4} \epsilon=2^{-\mid n_{i}-3} \epsilon
$$

which tends to zero as $n \rightarrow-\infty$ (i.e. as $x \rightarrow a+0)$, while, if $n>0$ and $x_{n-1} \leqq x<x_{n}$,

$$
\begin{aligned}
0 & \leqq M_{0}(b)-F(b)-M_{0}(x)+F(x) \\
& <\sum_{\nu=n}^{\infty} 2^{-|n|-4} \epsilon=2^{-|n|-3} \epsilon
\end{aligned}
$$

which tends to zero as $n \rightarrow \infty$ (i.e. as $x \rightarrow b-0$ ). Thus $M_{0}(x)-F(x)$ is continuous, relative to $(a, b)$, at $x=a$ and $x=b$, and hence it follows from $(2 \cdot 2)$ that $M I_{0}(x)$ is $C_{\lambda}$-continuous in $a \leqq x \leqq b$. So $M_{0}(x)$ satisfies (1) and (2), and it is easily seen that (3) is satisfied with $\frac{1}{4} \epsilon$ in place of $\epsilon$, while (4) and (5) are satisfied except possibly at $x=a$ and $x=b^{1}$.

${ }^{1}$ If in the definition of a $C_{\lambda} P$ integral an exceptional null set $S$ is allowed in condition (4) and an exceptional enumerable set $E$ in condition (5), the rest of the proof, which shows explicitly how to "remove" an exceptional point, may be omitted. Burkill, 3, 317, 5, 46, 6, 223, showed, in the case $0<\lambda \leqq 1$, that such a definition is possible, but did not show explicitly (in the case of the set $E$ ) that the scope of the integral is not increased thereby. In the general case the set $S$ is easily reduced to the set $E$ by the addition to $M(x)$ of an appropriate function with infinite derivates at the points of $S$ [Burkill, 2, 274, 3, 317], while the set $E$ may be removed by repetition of the argument given here [or by repetition of Miss Grimshaw's argument in the case $\lambda=1 \mathrm{j}$. Thus it may be shown that the scope of the $C_{\lambda} P$ integral $(\lambda>0)$ is not increased by the general definition. I am indebted to Miss W. L. C. Sargent for helping me to clear up this point. 
Let $z_{1}(x), z_{2}(x)$ be defined so that (i) $z_{1}(a)=0$, (ii) $z_{1}(x)$ is continuous and steadily increasing for $a \leqq x \leqq b$, (iii) $z_{1}(b)<\frac{1}{4} \epsilon$, (iv) $z_{1}(x) \geqq(\lambda+1)\left|C_{\lambda}\left(M_{0}, a, x\right)\right|$ in some interval $\left(a, a+\eta_{1}\right)$, (v) $z_{1}(x) /(x-a)$ is steadily decreasing in $\left(a, a+\eta_{1}\right)$, while (vi) $z_{2}(a)=0$, (vii) $z_{2}(x)$ is continuous and steadily increasing in $a \leqq x \leqq b$, (viii) $0<z_{2}(b)<\frac{1}{4}, \quad$ (ix) $z_{2}(x) \leqq z_{2}(b)-(\lambda+1)\left|C_{\lambda}\left(M_{0}, b, x\right)-M_{0}(x)\right|$ in some interval $\left(b-\eta_{2}, b\right), \quad(\mathrm{x}) \quad\left\{z_{2}(x)-z_{2}(b)\right\} /(x-b) \quad$ is steadily increasing in $\left(b-\eta_{2}, b\right)^{1}$.

Then, for $0<h<\eta_{1}$, it follows from ( $v$ ) that

$$
\begin{aligned}
\frac{C_{\lambda}\left(z_{1}, a, a+h\right)}{h /(\lambda+1)} & =\frac{\lambda(\lambda+1)}{h^{2}} \int_{0}^{h}\left(1-\frac{u}{h}\right)^{\lambda-1} z_{1}(a+u) d u \\
& \geqq \frac{\lambda(\lambda+1) z_{1}(a+h)}{h^{3}} \int_{0}^{h}\left(1-\frac{u}{h}\right)^{\lambda-1} u d u \\
& =\frac{z_{1}(a+h)}{h} .
\end{aligned}
$$

and hence from (iv) that

$$
\begin{aligned}
\lim _{h \rightarrow+0} & \frac{C_{\lambda}\left(M_{0}+z_{1}, a, a+h\right)}{h /(\lambda+1)} \geqq \lim _{h \rightarrow+0}\left\{\frac{z_{1}(a+h)}{h}+\frac{C_{\lambda}\left(M_{0}, a, a+h\right)}{h /(\lambda+1)}\right\} \\
& \geqq \lim _{h \rightarrow+0} \frac{(\lambda+1)}{h}\left\{\left|C_{\lambda}\left(M_{0}, a, a+h\right)\right|+C_{\lambda}\left(M_{0}, a, a+h\right)\right\} \\
& \geqq 0
\end{aligned}
$$

while, for $-\eta_{2}<h<0$, it follows from (x) that

$$
\begin{aligned}
\frac{C_{\lambda}\left(z_{2}, b, b+h\right)-z_{2}(b)}{h /(\lambda+1)} & =\frac{\lambda(\lambda+1)}{h^{2}} \int_{0}^{h}\left(1-\frac{u}{h}\right)^{\lambda-1}\left\{z_{2}(b+u)-z_{2}(b)\right\} d u \\
& \geqq \frac{z_{2}(b+h)-z_{2}(b)}{h},
\end{aligned}
$$

1 For instance, if $\eta_{1}$ is chosen so that $(\lambda+1)\left|C_{\lambda}\left(M_{0}, a, x\right)\right|<\frac{1}{4} \epsilon$ in $\left(a, a+\eta_{1}\right)$, we may take

$$
z_{1}(x)=(\lambda+1)(x-a) \underset{x \leqq t \leq a+\eta_{1}}{\overline{\text { bound }}}\left\{\frac{1}{t-a} \underset{a \leqq u \leqq t}{\overline{\text { bound }}}\left|C_{\lambda}\left(M I_{0}, a, u\right)\right|\right\}
$$

in $\left(a, a+\eta_{1}\right)$, and $z_{1}(x)$ constant in $\left(a+\eta_{1}, b\right)$, while if $\eta_{2}$ is chosen so that

$$
(\lambda+1)\left|C_{\lambda}\left(M_{0}, b, x\right)-M_{0}(b)\right|<\ddot{z}_{2}(b)
$$

in $\left(b-\eta_{2}, b\right)$, where $:_{2}(b)$ satisfies (viii), we may take

$$
z_{2}(x)=z_{2}(b)-(\lambda+1)(b-x) \underset{b-\eta_{i} \leqq t \leq x}{\overline{\text { bound }}} \frac{1}{b-t}\left\{\overline{\text { bound }} ; C_{\lambda}\left(M_{0}, b, u\right)-M_{0}(b) \mid\right\}
$$

in $\left(b-\eta_{2}, b\right)$, and $\tilde{z}_{2}(a)$ linear in $\left(a, b-\eta_{2}\right)$. 
and hence from (ix) that

$$
\begin{aligned}
& \lim _{h \rightarrow-0} \frac{C_{\lambda}\left(M_{0}+z_{2}, b, b+h\right)-M_{0}(b)-z_{2}(b)}{h /(\lambda+1)} . \\
& \geqq \lim _{h \rightarrow-0}\left\{\frac{C_{\lambda}\left(M_{0}, b, b+h\right)-M_{0}(b)}{h /(\lambda+1)}+\frac{z_{2}(b+h)-z_{2}(b)}{h}\right\} \\
& \left.\geqq \lim _{h \rightarrow-0} \frac{(\lambda+1)}{h}\left\{C_{\lambda} M_{0}, b, b+h\right)-M_{0}(b)-\left|C_{\lambda}\left(M_{0}, b, b+h\right)-M_{0}(b)\right|\right\} \\
& \geqq 0 .
\end{aligned}
$$

If we now define

$$
M(x)=M_{0}(x)+z_{1}(x)+z_{2}(x)+\frac{1}{8} \epsilon\left(\frac{x-a}{b-a}\right)^{\frac{1}{2}}+\frac{1}{8} \epsilon\left\{1-\left(\frac{b-x}{b-a}\right)^{\frac{1}{2}}\right\}
$$

for $a \leqq x \leqq b$, we see that $M(x)$ satisfies conditions (1)-(5).

\section{Applications.}

I. Theorem $\mathrm{A}$ has been used elsewhere ${ }^{1}$ to prove that a function integrable in the $C_{\lambda} L$ sense (Cesàro-Lebesgue, $\lambda \geqq 0$ ) is also integrable in the $C_{\lambda} P$ sense, i.e. that the $C_{\lambda} L$ integral is included in the $C_{\lambda} P$ integral.

II. Miss Sargent has recently defined ${ }^{2}$ a $C_{\lambda} D$ integral (Special Cesàro-Denjoy, $\lambda$ =integer), and used Theorem $\mathbf{A}$ in proving that it is equivalent to the $C_{\lambda} P$ integral.

\footnotetext{
1 Bosanquet, 1.

2 Sargent, 9.
}

\section{REFERENCES.}

1. L. S. Bosanquet, "A solution of the Cesàro summability problem for successively derived Fourier series," Proc. London Math. Soc. (2), 46 (1940), 270-289.

2. J. C. Burkill, "The approximately continuous Perron integral," Math. Zeitschrift, 34 (1932), 270-278.

3. — "The Cesàro-Perron integral," Proc. London Math. Soc. (2), 34 (1932), 314-322.

4. - "The Cesàro-Perron scale of integration," Proc. London Math. Soc. (2), 39 (1935), 541-552. 
5. J. C. Burkill, "The expression of trigonometrical series in Fourier form," Journal London Math. Soc., 11 (1936), 43-48.

6. __ " "Fractional orders of integrability," Journal London Math. Soc., 11 (1936), 220-226.

7. M. E. Grimshaw, "The Cauchy property of the generalised Perron integrals," Proc. Cambridge Phil. Soc., 30 (1934), 15-18.

8. S. Saks, Theory of the integral (Warsaw, 1937).

9. W. L. C. Sargent, "A descriptive definition of Cesàro-Perron integrals." Unpublished.

\section{University College, LONDON.}

